\title{
Frontières
}

\section{Bibliographie indicative sur l'aide médicale à mourir}

\section{Marie-Luce Delfosse et Jocelyne Saint-Arnaud}

Volume 24, numéro 1-2, automne 2011, printemps 2012

URI : https://id.erudit.org/iderudit/1013099ar

DOI : https://doi.org/10.7202/1013099ar

Aller au sommaire du numéro

Éditeur(s)

Université du Québec à Montréal

ISSN

1916-0976 (numérique)

Découvrir la revue

Citer ce document

Delfosse, M.-L. \& Saint-Arnaud, J. (2011). Bibliographie indicative sur l'aide médicale à mourir. Frontières, 24(1-2), 131-133.

https://doi.org/10.7202/1013099ar d'utilisation que vous pouvez consulter en ligne.

https://apropos.erudit.org/fr/usagers/politique-dutilisation/ 


\section{BIBLIOGRAPHIE INDICATIVE SUR L'AIDE MÉDICALE À MOURIR \\ Marie-Luce Delfosse et Jocelyne Saint-Arnaud}

ABBOTT, K.H., J.G. SAGO, C.M. BREEN, A.P. ABERNETHY et J.A. TULSKY (2001). « Families looking back: one year after discussion of withdrawal or withholding of life-sustaining support», Critical Care Medicine, vol. 29, n 1, p. 197-201.

AUMONIER, N., B. BEIGNIER et P. LETELLIER (2010). L'euthanasie, Paris, P.U.F., collection «Que sais-je ?", 3595 (5 éd. mise à jour).

AURENCHE S. (DIR.) (2003). La mort devant soi. Euthanasie, des clés pour un débat, Paris, Éditions Autrement.

BÉLAND J.P. (DIR.) (2008). Mourir dans la dignité? Soins palliatifs ou suicide assisté, un choix de société, Québec, Presses de l'Université Laval.

BOISVERT, M. et S. DANEAULT (2010). Être ou ne pas être. Débat sur l'euthanasie, Montréal, Les Éditions Parallèles, $159 \mathrm{p}$.

BREEN, C.M., A.P. ABERNATHY., H. ABBOTT et J.A. TULSKY (2001). "Conflict associated with decisions to limit life-sustaining treatment in intensive care units ", Journal of General Internal Medicine, vol. $16, n^{\circ}$ 5, p. 283-289.

BUITING, H.M., A. VAN DER HEIDE, B.D. ONWUTEAKE-PHILIPSEN, M.L. RURUP, J.A.C. RIETJENS, G. BOORSBOOM, P.J. VAN DES MAAS et J.J.M. VAN DELDEN (2010). "Physicians' labelling of end-of-life practices: A hypothetical case study ", Journal of Medical Ethics, vol. 36, p. 24-29.

CAILLAVET, H. (2002). Comment mourir dans la dignité, Nantes, Pleins feux.

CASSELL, E.J. (1991). "Recognizing suffering". Hastings Center Report, vol. 21, n 3, p. 24-31.

CASSIERS, L. (2011). Ni ange, ni bête: essai sur l'éthique de I'homme ordinaire, Paris, Cerf, collection «Recherches morales. Nouvelle série».

CHAMBAERE, K., J. COHEN, S. STERCKX, F. MORTIER et al. (2011). "Palliative sedation in Flemish palliative care units", Journal of Pain and Symptom Management, vol. 41, $\mathrm{n}^{\circ} 6$.

CHAO, D.V.K., N.Y. CHAN et W.Y. CHAN (2001). "Euthanasia revisited», Family Practice vol. 19, $n^{\circ} 2,128-134$

CHÂTELET, N. (2005). La dernière leçon, $n^{\circ} 1380$,

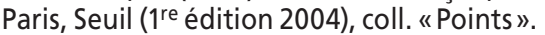

CHAUSSOY, F. (2004). Je ne suis pas un assassin, Paris, Oh! Editions.

CLAESSENS, P., J. MENTEN, P. SCHOTMANS, B. BROECKAERT (2008). "Palliative Sedation: a Review of the Research Literature ", Journal of Pain and Symptom Management, vol. 36, $\mathrm{n}^{\circ} 3$, p. 310-333.

COHEN-ALMAGOR, R. (2009). "Belgian euthanasia law: a critical analysis ", Journal of Medical Ethics, vol. 35, p.436-439.

COLLECTIF (2008). Les directives anticipées comparaison internationale des points de vue juridique et éthique, $\mathrm{n}^{\circ}$ spécial du Bulletin de la Société des sciences médicales du Grand Duché de Luxembourg, $n^{\circ} 3$.
COLLECTIF (2012), «Euthanasie et soins palliatifs en Belgique», La revue des soins palliatifs en Wallonie, en ligne, <soinspalliatifs.be>.

COMITÉ CONSULTATIF DE BIOÉTHIQUE DE BELGIQUE, Avis n 1 du 12 mai 1997 concernant l'opportunité d'un règlement légal de l'euthanasie.

COMITÉ CONSULTATIF DE BIOÉTHIQUE DE BELGIQUE, Avis n 9 du 22 février 1999 concernant l'arrêt actif de la vie des personnes incapables d'exprimer leur volonté.

COMMISSION FÉDÉRALE DE CONTRÔLE ET D'ÉVALUATION DE L'EUTHANASIE (BELGIQUE), Brochure à l'intention du corps médical, en ligne, <http://www.health.fgov.be/euthanasie>.

COMMISSION FÉDÉRALE DE CONTRÔLE ET D'ÉVALUATION DE L'EUTHANASIE (BELGIQUE) (ANNÉES 2008 ET 2009), Quatrième rapport aux chambres législatives, en ligne, <www.ieb-eib. org/nl/pdf/rapport-euthanasie.pdf>.

COMMISSIONS RÉGIONALES DE CONTRÔLE DE L'EUTHANASIE (CRCE), Rapport 2007, Rapport 2008, Rapport 2009, en ligne, <www.ieb.eib.org/ $\mathrm{fr} / \mathrm{pdf} / \mathrm{rapport}$-euthanasie-pays-bas-2009-en-fr$p d f>$.

COMMISSION SPÉCIALE DE L'ASSEMBLÉE NATIONALE SUR LA QUESTION DE MOURIR DANS LA DIGNITÉ (2010). Mourir dans la dignité. Document de consultation, Gouvernement du Québec, mai, $37 \mathrm{p}$.

COMTE-SPONVILLE, A., M. DE HENNEZEL et A. KAHN, (sous la direction de Alain Houziaux) (2004). Doit-on légaliser l'euthanasie? Ivry-surSeine (Val-de-Marne), Éd. de l'Atelier, collections "Questions de vie».

CONSEIL D'EUROPE, Études de législation, en ligne, <http://www.senat.fr/lc/lc109/lc1092. html>.

DANEAULT, S. (V. LUSSIER et S. MONGEAU, collab.) (2006). Souffrance et médecine, Québec, Presses de I'Université du Québec, 158 p.

DE HENNEZEL, M. (1996). Sens et valeur du temps qui précède la mort. In La fin de la vie, qui en décide? Forum Diderot, Paris, Presses universitaires de France, p. 19-28.

DE HENNEZEL, M. (2004). Le souci de l'autre. Poche, Paris, Robert Laffont.

DELIENS, L. et al. (2000). «End-of-life decisions in medical practice in Flanders, Belgium: a nationwide survey ", The Lancet, n 356, p. 1806-1811.

DENIER, Y., B. DIERCKX DE CASTERLEE, N. DE BAL et C. GASTMANS (2009). "Involvement of nurses in the euthanasia care process in Flanders (Belgium): An exploration of two perspectives ${ }_{i}$ Journal of Palliative Care, vol. 25, n 4, p. 264-274.

D'HAENE, I., H. R.W PASMAN., L. DELIENS, J. BILSEN, F. MORTIER et R. VANDER STICHELE (2010). "End-of-life care policies in Flemish residential care facilities accommodating persons with intellectual disabilities", Journal of Intellectual Disability Research, vol. 54, n 12, décembre, p. 1067-1077.

D'HAENE, I., R. H. VANDER STICHELE, H. ROELINE W. PASMAN, N. VAN DEN NOORTGATE, J. BILSEN F. MORTIER et L. DELIENS (2009). "Policies to improve end-of-life decisions in Flemish hospitals: communication, training of health care providers and use of quality assessments", BMC Palliative Care, vol. 8, $\mathrm{n}^{\circ} 20$, en ligne, <http:// www.biomedcentral.com/1472-684X/8/20>.
DISTELMAN, W. (2005). Een waardig levenseinde, Antwerpen, Houtekiet, p. 1067-1077.

DUPONT, B.-M. (2011). D'un prétendu droit de mourir par humanité. L'euthanasie en question, Paris, François Bourin Éditeur.

EMANUEL, E.J., D.L. FLAIRCLAOUGH et L.L. EMANUEL (2000). "Attitudes and desires related to euthanasia and physician-assisted suicide among terminally ill patients and their caregivers", JAMA, vol. 284, n 19 , p. 2460-2468.

ENGLERT, M. (2010). «Le quatrième rapport de la commission fédérale de contrôle et d'évaluation de l'euthanasie est publié (Années 2008 et 2009) ", $A D M D$, Bulletin trimestriel, $3^{\mathrm{e}}$ trimestre 2010, n 117, p. 14-15.

ENGLERT, Y., J. VESTERGAARD, G. JAVASHVILI, G. KIKNADZE, B. BLASSZAUER, J. JM VAN DELDEN, D. SERRAO, A. BONDOLFI, S. MCLEAN, L. SNYDER, D. MARTY, E. GADD et P. MIERZEWKSKi (2004). Regard éthique: L'euthanasie. Volume II: Perspectives nationales et européennes, Strasbourg, Éditions du Conseil de l'Europe.

ÉQUIPE DE SOUTIEN DU RÉSEAU HOSPITALIER D'ANVERS, CAMPUS MIDDELHEIM (2008). Face à la mort. Récits d'euthanasie, trad. du néerlandais (Flandre) par Jeanne-Marie Degueldre, Bruxelles, Éditions Aden.

FERREL, B.R., D. NOVI, M.D. SULLIVAN, J. BANIA, M.Y. DUBOIS, M.C. GITLIN, D. HAMATY, A.. LEBOVITS, A.G. LIPMAN, P.M. LIPPE et J. LILVVICH (2001). "Ethical dilemmas in pain management», The Journal of Pain, vol. 2, n³, p. 171-180.

FERRY, L. et A. KAHN (2010). Faut-il légaliser I'euthanasie? Paris, Odile Jacob, collection "Penser la société ».

GANZINI, L., NELSON, H.D., SCHMIDT, T.A., KRAEMER, D.F., DELORIT, M.A., et LEE, M.A. (2000). "Physicians'experience with Oregon Death with Dignity Act ", The New England Journal of Medicine, vol. 342, n 8 , p. 557-563.

GENICOT, G. (2010). Droit médical et biomédical, Bruxelles, Larcier, "Collection de la Faculté de droit de Liège $"$.

GERVERS, S. (2007). «Selected Legislation and Jurisprudence. Evaluation of the Ducht on legislation euthanasia and assisted suicide », European Journal of Health Law, vol. 14, p. 369-379.

GRIFFITHS, J., H. WEYERS et M. ADAMS (2008). Euthanasia and Law in Europe, Oxford and Portland (Oregon), Hart Publishing, $595 \mathrm{p}$.

GUILLON, C. (2010). Le droit à la mort: suicide, mode d'emploi, ses lecteurs et ses juges, Paris, Éditions Hors commerce, 2004; $2^{\mathrm{e}}$ édition: Paris, IMHO, collection «Essais».

HADJISTAVROPOULOS, T. et K.D. CRAIG (2004). "Ethical issues for psychologists", Pain Psychological Perspectives. T. Hadjistavropoulos \& K.D. Craig (dir.). Maswah, New Jersey, Lawrence Erlbaum Associates Publishers, 327-344.

HAVE H. TEN (2005). End-of-Life Decision Making. A Cross-National Study, R.H. Blank \& J.C. Merrick (dir). Cambridge, The MIT Press.

HAVERKATE, I. A. VAN DER HEIDE, B. D. ONWUTEAKA-PHILIPSEN, P.J. VAN DER MAAS et G. VAN DER VAL (2001). "The emotional impact on physicians of hastening the death of a patient », The Medical Journal of Australia, vol. 175, p. 519-522. 
HERTOGH, C.M.P.M. (2009). «The role of advance directives as an aid to communication and shared decision-making in dementia», Journal of Medical Ethics, vol. 35, p. 100-103.

HICKS, T. (2000). «Ethical implications of pain management in a nursing home: A discussion", Nursing Ethics, vol. 7, n5, p. 392-398.

HINTERMEYER, P. (2003). Euthanasie, la dignité en question, Paris, Buffet/Chatel.

HIRSCH, E. (dir.) (2004). Face aux fins de vie et à la mort. Éthique et pratiques professionnelles au cœur du débat. Espace Éthique: Assistance publique et Hôpitaux de Paris, Paris, Vuibert.

HOUZIAUX, A. (sous la dir. de), avec la participation de A. Comte-Sponville, M. de Hennezel et A. Kahn (2004). Doit-on légaliser l'euthanasie?, Paris, Les éditions de l'Atelier/Les éditions ouvrières.

HUMBERT, V. (2003). Je vous demande le droit de mourir. Propos recueillis par F. Veille. Neuilly-surSeine, Éditions Michel Lafont.

IEDEREEN HEEFT RECHT OP EEN WAARDIG LEVENSEINDE, LEIFBLAD (journal du Levenseinde informatieforum) (forum belge en néerlandais sur la fin de vie), en ligne, <www.leif.be>.

JACQUEMIN, D. (2002). Bioéthique médecins et souffrance. Jalons pour une théologie de l'échec, Montréal, Mediapaul.

KIRK, P., I. KIRK et L.J. KRISTJANSON (2004). " "What do patients receiving palliative care for cancer and their families want to be told?" A Canadian and Australian qualitative study", British Medical Journal, 328, 1343-1350.

LABAYLE, D. (2009), Pitié pour les hommes. L'euthanasie: le droit ultime, Paris, Stock, Collection «Parti pris».

LALLEMAND, R. et P. de LOCHT (2001). L'euthanasie, Bruxelles, EVO.

LEGUAY, C. (2005). Respecter la vie, disposer de sa mort! Pour une loi Vincent Humbert, Paris, L'Harmattan.

LEMIENGRE, J. (2010). Written ethics policies on euthanasia in Flemish hospitals and nursing homes: an empirical-ethical study on development, content and impact, Leuven, Leuven University Press, collection "Acta biomedica Lovaniensia ", $490 \mathrm{p}$.

LEMIENGRE, J. , C. GASTMANS, P. SCHOTSMANS et B. DIERCKX de CASTERLÉ, (2010), "Impact of Written Ethics Policy on Euthanasia From the Perspective of Physicians and Nurses: A Multiple Case Study in Hospitals ", AJOB Primary Research, $1: 2$, p. 49-60.

LEMIENGRE, J., B. DIERCKX de CASTERLE', Y. DENIER, P. SCHOTSMANS et C. GASTMANS (2008). "How do hospitals deal with euthanasia requests in Flanders (Belgium)? A content analysis of policy documents", Patient Education and Counseling, 71, p. 293-301.

LEMIENGRE, J., B. DIERCKX de CASTERLE, G. VERBEKE, K. VAN CRAEN et P. SCHOTSMANS, C. GASTMANS (2008), "Ethics policies on euthanasia in nursing homes: A survey in Flanders, Belgium ", Social Science \& Medicine, 66, p. 376-386.

LEMIENGRE， J., B. DIERCKX de CASTERLÉ, G. VERBEKE, C. GUISSON, P. SCHOTSMANS et C.
GASTMANS (2007). «Ethics policies on euthanasia in hospitals-A survey in Flanders (Belgium)", Health Policy, 84, p. 170-180.

LEMIENGRE，J., B. DIERCKX de CASTERLE, Y. DENIER, P. SCHOTSMANS, C. GASTMANS (2009). "Content analysis of euthanasia policies of nursing homes in Flanders (Belgium)", Med. Health Care and Philos., 12, p. 313-322

LESAGE, P. et R. K. PORTENOY (2001). «Ethical challenges in the care of patients with serious illness ", Pain Medicine, n², vol 2, p. 121-130.

LETELLIER, P. et al. (2003). L'euthanasie. Volume 1: Aspects humains et éthiques, Strasbourg, Éditions du Conseil de l'Europe.

LEWIS, P. (2009). "Euthanasia in Belgium Five Years After Legalisation ", European Journal of Health Law, 16, p. 125-138.

LIBOVITS, A. (2001). "Ethics and Pain: Why and for Whom ?", Pain Medicine, vol. 2, n² 2, p. 92-96.

LOEWY, R.S. (2004). "Hastening death by selective disclosure of treatment options Beneficience or "Euthanasia by deception" ?", Health Care Analysis, vol. 12, n³, p. 241-250.

MAESSEN, M., J.H. VELDINK, B.D. ONWUTEAKAPHILIPSEN, J.M. DE VRIES, J.H.J. WOKKE, G. VAN DER WAL et L.H. VAN DEN BERG (2009). «Trends and determinants of end-of-life practices in ALS in the Netherlands", Neurology, vol. 73, $n^{\circ} 20$, p. 954-961.

MALTONI, M., C. PITTURI, E. SCARPI, L. PÎCCINI, F. MARTINI, P. TRUCI, L. MONTANARI, O. WWNANNI et D. AMADORI (2009). "Palliative sedation therapy does not hasten death: Results from a prospective multicenter study ", Annals of Oncology, vol. 20, 1163-1169.

MARCOUX, I. (2007). "Dans quelles conditions est-il acceptable ou non de mourir par euthanasie?", Frontières, vol. 20, n 1, p. 69-75.

MARIN, I. (2003). "Un don paradoxal?", dans La mort devant soi. Euthanasie, des clés pour un débat, sous la direction de S. Aurenche, Paris, Les Éditions Autrement, p. 81-91.

NORWOOD, F., G. KIMSMA et M.P. BATTIN (2009). "Vulnerability and the "slippery slope" at the end of life: A qualitative study of euthanasia, general practice and home death in the Netherlands", Family Practice, vol. 26, nº 6, p. 472-480.

ORR, R.D. (2001). «Pain management rather than assisted suicide: The ethical high ground", Pain Medicine, vol. 2, n², p. 131-137.

POUSSET, G., F. MORTIER, J. BILSEN, J. COHEN, L. DELIENS (2010). «Attitudes and practices of physicians regarding physician-assisted dying in minors", Archives of Disease in Childhood, en ligne depuis le 23 juin.

QUILL, T.E. et I.R. BYOCK (2000). "Responding to intractable terminal suffering: The role of terminal sedation and voluntary refusal of food and fluids. Position Paper of the American College of Physicians \& American Society of Internal Medicine ». Annals of Internal Medicine, vol. 132, n5, p 408-414.

RACHELS, J. (1986). The End of Life: Euthanasia and Morality, Oxford, Oxford University Press.

RAUS, K., S. STERCKX et F. MORTIER (2011). «Is continuous sedation at the end of life an ethically preferable alternative to physician-assisted suicide?", The American Journal of Bioethics, vol. $11, n^{\circ} 6$, p. 32-40.
REUZEL, R.P.B., G.J. HASSELAAR, K.C.P. VISSERS, G.J. VAN DER BILT, J.M.M GROENEWOUD, B.J.P CRUL (2008). «Inappropriateness of using opioids for end-stage palliative sedation: A Dutch study ». Palliative Medicine, vol. 22, p. 641-646.

RIETJENS, J., J. VAN DELDEN, B. ONWUTEAKAPHILIPSEN, H. BUITING, P. VAN DER MASS et A. VAN DER HEIDE (2008). "Continuous deep sedation for patients nearing death in the Netherlands: Descriptive study ", British Medical Journal on Line, retiré du site BMJ.com le 8 février 2010.

SAINT-ARNAUD, J. (2006). "Enjeux éthiques reliés au consentement substitué dans le cas de l'arrêt de traitements maintenant la vie pour des personnes âgées en dialyse rénale et des bébés très prématurés ou lourdement handicapés à la naissance", Actes du XVIe Congrès mondial de droit médical, tenu à Toulouse en août 2006, Les Études hospitalières, vol. 2, p. 817-824.

SAINT-ARNAUD, J. (2010). La décriminalisation, la légalisation et l'institutionnalisation des pratiques d'euthanasie: analyse de la question en fonction des théories et des principes éthiques, de l'examen des dérives possibles selon la pratique légalisée dans les Pays-Bas et enfin, du contexte des soins de fin de vie au Québec. Mémoire présenté à la Commission parlementaire sur le droit de mourir dans la dignité, Québec, 17 février 2010, disponible sur le site de l'Assemblée nationale du Québec

SCHUMACHER, B-N. (2011). Quand cesse-t-on de vivre?: pour une définition de la mort humaine, Nantes, Ed. C. Defaut.

SENNET, B. (2003). "Briser la loi du silence». Propos recueillis par $\mathrm{S}$. Aurenche, dans La mort devant soi. Euthanasie, des clés pour un débat, sous la direction de $S$. Aurenche, Paris, Les éditions Autrement, p. 73-80.

SMETS, T., J. BILSEN, J. COHEN, M. L. RURUP et L. DELIENS (2009). «Legal euthanasia in Belgium. Characteristics of all reported euthanasia cases", Medical Care, vol. 47, $\mathrm{n}^{\circ} 12$

SMETS, T., J. COHEN, J. BILSEN, Y. VAN WESEMAEL, M. L. RURUP et L. DELIENS (2011). "Attitudes and experiences of belgian physicians regarding euthanasia practice and the euthanasia law", Journal of Pain and Symptom Management, vol. 41, n³, p. 580-593.

SCEUR LÉONTINE (1993). Au nom de la vie. Les soins palliatifs: éthique et témoignage, trad. du néerlandais par Guy Verwacht, Gembloux, Duculot.

STEINHAUSER, K.E., E.C. CLIPP, M. MCNEILLY, N.A. CHRISTAKIS, L.M. MCINTYRE et J.A. TULSKY (2000). "In search of a good death: Observations of patients, families, and providers", Annals of Internal Medicine, vol. 132, n 10, p. 825-832.

SWARTE, N. B., M. L. VAN DER LEE, J. G. VAN DER BOM, J. VAN DEN BOUT et A. P. M. HEINTZ, "Effects of euthanasia on the bereaved family and friends: a cross sectional study ", British Medical Journal, vol. 327, nº 189.

TACK, S. (2009). "Legal aspects of end-of-life decision policies in health care institutions", Med. Law, vol. 28, nº 4, p. 725-738.

TACK, S. (2011). "Can hospitals prohibit euthanasia? An analysis from a European human rights perspective ", Med. Law, vol. 30, n² 2, p. 295-306. 
VAN BRUCHEM-VAN DE SCHEUR, G.G., A.J.G. VAN DER AREND, H.H. ABU-SAAD, F.C.B. VAN WIJMEN, C. SPREEUWEBER et R. H. J. TER MEULEN (2008). "Alleviation of pain and symptoms with a life-shortening intention ", Nursing Ethics, vol. 15, $n^{\circ} 5$, p. 682-695.

VAN DEN BLOCK, L., R. DESCHEPPER, J. BILSEN, N. BOSSUYT, V. VAN CASTEREN, L. DELIENS (2009). "Euthanasia and other end of life decisions and care provided in final three months of life: nationwide retrospective study in Belgium ", British Medical Journal, vol. 339, b2772.

VAN DER GEEST et A. M. NIEKEMP (2003). "Ageism and euthanasia in the Netherlands: Questions and conjectures", Mortality, vol. 8, $\mathrm{n}^{\circ} 3$, p. 296-304.

VAN DER HEIDE, A., B. D. ONTWUTEAKA-PHILIPSEN, M. L. RURUP, H.M. BUITING, J.J.M. VAN DELDEN et al. (2007). "End-of-life practices in the Netherlands under the Euthanasia Act ", The New England Journal of Medicine, vol. 356, n 19, p. 1957-1965.

VAN DER MASS, P.J., G. VAN DER WAL, H. HAVERKATE et al. (1996). "Physician assisted suicide and other medical practices involving the end of life in the Netherlands 1990-1995 ", The New England Journal of Medicine, $n^{\circ} 335$, p. 1900-1705.

VAN MARWIJJK, H., I. HAVERKATE, P. VAN ROYEN et A.M. THE (2007). "Impact of euthanasia on primary care physicians in the Netherlands", Palliative Medicine, vol. 21, p. 609-614.

VAN WESEMAEL, Y., J. COHEN, J. BILSEN, T. SMETS, B. ONWUTEAKA-PHILIPSEN, L. DELIENS (2011), "Process and outcomes of euthanasia requests under the Belgian act on euthanasia: a nationwide survey", Journal of Pain and Symptom Management, vol. 42, n5, p.721-733.

VERHAGEN, A.A.E., J.H.H.M. DORSCHEIDT, B. ENGELS, J.H. HUBBEN et P.J. SAUER (2009). «Endof-life decisions in Dutch neonatal intensive care units?", Archives of Pediatrics \& Adolescent Medicine, vol. 163, n 10 , p. 895-901.

VERHAGEN, E., et P.J.J. SAUER (2005). "The Groningen protocol: Euthanasia in severely ill newborns", The New England Journal of Medicine, vol. 352, n 10, p. 952-962.

VIG, E. et R.A. PEARLMAN (2004). " Good and bad dying from the perspective of terminally ill men ", Archives of Internal Medicine, vol. 164, $n^{\circ}$ 9, p. 997-981.

WAY, J., A.L. BACK et J.R. CURTIS (2001). "Withdrawing life support and resolution of conflict with families ", British Medical Journal, vol. 325, p. 1342-1345.

WEIJER, C. (2005). "A death in the family. Reflections on the Terri Schiavo case ", Canadian Medical Association Journal, vol. 172, nº 9, p. 1197-1198.

WEISSMAN, D.E. (2004). "Decision making at a time of crisis near the end of life ", JAMA, vol. 292, p. 14 , p. $1738-1743$.

ZITTOUN, R. (2007). La mort de I'autre. Une introduction à l'éthique clinique, Paris, Dunod.

\section{NOUVELLES PARUTIONS - ÉTUDES SUR LA MORT}

Serge Gariépy,

bibliothécaire de référence,

Bibliothèque centrale, UQAM.

ABBEY, Jenny (2011). Facing Death and Dying in Dementia Care: A Person-Centred and Palliative Approach, Londres, Jessica Kingsley.

AMEISEN, Jean-Claude (dir.) (2010). Qu'est-ce que mourir? Paris, Éd. le Pommier.

BACHAND, Sarah et Caroline LABRIE (2011). Au-delà des mots: recueil sur le deuil périnatal, Québec, Publications du Québec.

BALK, Charles A. et David E. CORR (2010). Children's Encounters with Death, Bereavement, and Coping, New York (NY), Springer.

BÉLIVEAU, Richard (2010). La mort: mieux la comprendre et moins la craindre pour mieux célébrer la vie, Montréal, Trécarré.

BOISVERT, Marcel et Serge DANEAULT (2010). Être ou ne plus être: débats sur l'euthanasie, Montréal, Éditions Voix parallèles.

BRYANT, Clifton D. (2003). Handbook of Death and Dying, Londres, Sage Publications.

CARTRON, Isabelle (2010). De corps en corps: Traitement et devenir du cadavre, Bordeaux, MSHA.

COWAN, Richard et Michael MCQUELLON (2010). The Art of Conversation Through Serious IIIness: Lessons for Caregivers, Oxford (UK), Oxford University Press.

CRETTAZ, Bernard (2010). Cafés mortels: sortir la mort du silence, Genève, Labor et Fidès.

DABOUIS, Gérard (2010). La mort: journées de la Maison des sciences de l'homme Ange-Guépin, Paris, L'Harmattan.

DI FOLCO, Philippe (dir.) (2010). Dictionnaire de la mort, Paris, Larousse.

DUPONT, Bernard-Marie (2011). D'un prétendu droit de mourir par humanité: l'euthanasie en question, Paris, François Bourin.

FOURNIER, Édith (2010). J'ai commencé mon éternité: survivre au déclin de l'autre, Montréal, Éditions de l'Homme.

GREY, Robin (2010). Bereavement, Loss and Learning Disabilities: A Guide for Professionals and Carers, Londres, Jessica Kingsley.

GUILLAUD, Lauric (2010). Le retour des morts: imaginaire, science, verticalité, Pertuis, Rouge Profond.

HESS, Geneviève (2010). Accompagnement d'une mère en fin de vie: journal à quatre mains, Paris, Téraèdre.

KRAUS, Barbara et Frances MONROE (2010). Brief Interventions with Bereaved Children, Oxford (UK), Oxford University Press.
MONBOURQUETTE, Jean et Isabelle D'ASPREMONT (2011). Excusez-moi, je suis en deuil, Montréal, Novalis.

NORWOOD, Frances (2010). Mourir un acte de vie: prévenir la mort sociale par la discussion pré-euthanasie et les soins de fin de vie, Québec, Presses de l'Université Laval.

ONANA, Paul-Bienvenu (2010). L'Herméneutique platonicienne de la mort, Paris, L'Harmattan.

PROKOVYEV, Luka (2010). Diagnosing Death: Issues, Ethics and Questions in Death Determinations, New York (NY), Nova Science Publishers.

RICHARD, Louis-André et Michel L'HEUREUX (2011). Plaidoyer pour une mort digne, Québec, Presses de l'Université Laval.

SALAMON, Michel (2010). La mort interdite, Limoges, Pulim.

SCHUMACHER, Bernard N. (2011). Death and Mortality in Contemporary Philosophy, New York (NY), Cambridge University Press.

SOCIÉTÉ DE THANATOLOGIE (FRANCE) (2009). Deuils et grand âge: peut-on apprendre à vieillir? Le Bouscat (France), L'Esprit du temps.

THOMAS, Louis-Vincent (2010). Mort et pouvoir, Paris, Payot et Rivages.

VASSY, Carine (dir.) (2010). Enregistrer les morts, identifier les surmortalités: une comparaison Angleterre, États-Unis et France, Rennes, Presses de l'EHESP.

WEBB, Nancy Boyd (2010). Helping Bereaved Children: A Handbook for Practitioners, New York, Guilford Press.

YOUNG, Terence (2011). Mourir sur ordonnance: un père enquête sur l'industrie pharmaceutique, Montréal, Écosociété. 